\title{
Arborescences
}

Revue d'études françaises

\section{Identités linguistiques, langues identitaires : synthèse}

\section{Anne-Marie Brousseau}

\section{Numéro 1, mars 2011}

Identités linguistiques, langues identitaires : à la croisée du prescriptivisme et du patriotisme

URI : https://id.erudit.org/iderudit/1001938ar

DOI : https://doi.org/10.7202/1001938ar

Aller au sommaire du numéro

\section{Éditeur(s)}

Département d'études françaises, Université de Toronto

ISSN

1925-5357 (numérique)

Découvrir la revue

Citer cet article

Brousseau, A.-M. (2011). Identités linguistiques, langues identitaires : synthèse. Arborescences, (1), 0-0. https://doi.org/10.7202/1001938ar

\section{Résumé de l'article}

Cet article propose une synthèse des huit articles de ce numéro, qui sont issus du colloque « Prescriptivism(e) \& Patriotism(e): du nationalisme à la mondialisation » tenu à Toronto les 17-19 août 2009. Pour faire bon accueil à une diversité de lectrices et lecteurs intéressés par les études françaises, cette synthèse situe les articles dans la grande conversation sociolinguistique qui porte sur les rapports entre langue et identité. Elle définit d'abord les deux concepts qui ont inspiré ce numéro : 1) le prescriptivisme, en rapport à une série de notions qui y sont reliées (norme, surnorme, bon usage, variation linguistique, marché linguistique) ; et 2) le patriotisme, en fonction de son lien essentiel avec l'identité (construction identitaire, rapport à l'autre, attitudes linguistiques, prestige manifeste, prestige latent). Elle résume ensuite chacun des articles, regroupés en trois parties, abordant chacune une facette de la dynamique patriotisme-prescriptivisme. La première partie traite du décalage entre les normes et les usages linguistiques et la perception de ces normes et de ces usages. La deuxième partie aborde la question de la vitalité linguistique, de l'érosion linguistique, de l'aménagement des langues et de leur revitalisation. La troisième partie montre les relations intestines, parfois même symbiotiques, entre le prescriptivisme et le patriotisme.
Tous droits réservés @ Département d'études françaises, Université de Toronto, 2011
Ce document est protégé par la loi sur le droit d'auteur. L'utilisation des services d'Érudit (y compris la reproduction) est assujettie à sa politique d'utilisation que vous pouvez consulter en ligne.

https://apropos.erudit.org/fr/usagers/politique-dutilisation/ 


\title{
IDENTITÉS LINGUISTIQUES, LANGUES IDENTI'TAIRES
}

\author{
Synthèse
}

\author{
Anne-Marie Brousseau \\ Université de Toronto
}

\section{Introduction}

Les articles présentés dans ce numéro ont vu le jour lors du colloque «Prescriptivism(e) \& Patriotism(e): du nationalisme à la mondialisation ${ }^{1}$, qui a accueilli plus d'une cinquantaine de communications à Toronto les 17-19 août 2009. Ce colloque visait à regrouper des chercheurs d'horizons divers, reflétant ainsi la diversité qui caractérise sa ville-hôte, Toronto : il s'agissait donc d'un colloque bilingue (français et anglais), multiculturel (avec des participantes et participants ${ }^{2}$ venus des cinq continents) et interdisciplinaire (regroupant lexicographie, linguistique historique, éducation, analyse de discours, sociolinguistique et anthropologie).

Le présent numéro ne constitue pas les actes du colloque à proprement parler, mais plutôt une sélection des textes issus du colloque, qui sera bientôt complétée par une deuxième sélection à paraitre dans la revue Multilingual Matters. Cette sélection-ci est surtout francophone (six articles en français), mais aussi francocentrique (deux articles en anglais portant l'un sur le français et l'autre sur un créole à base lexicale française). Les textes sont regroupés en trois parties, abordant chacune une facette de la dynamique patriotisme-prescriptivisme. Chacune de ces facettes se distingue des autres à plus d'un niveau: par les questions qu'elle soulève, par les réponses qu'elle propose, par les approches méthodologiques qu'elle utilise, par les communautés et les productions linguistiques qu'elle examine.

Du Québec au Manitoba, des Caraïbes à la Nouvelle-Angleterre, du Moyen-Orient au Cameroun, en passant par la France d'aujourd'hui et d'hier, les articles recueillis brossent les portraits

\footnotetext{
${ }^{1}$ Ce colloque a bénéficié d'une subvention du Conseil de recherches en sciences humaines du Canada, \# 646-2008-1115 (concours novembre 2008).

2 Bien que cette norme n'ait pas été imposée à l'ensemble de ce numéro, j’ai adopté les procédés de féminisation des textes préconisés au Québec et au Canada français. Voir, par exemple, http://www.onterm.gov.on.ca/feminisation.html.
} 
de langues ${ }^{3}$ identitaires diverses : français québécois, français manitobain, «patois » de Sainte-Lucie, français franco-américain, hébreu, français hexagonal des nouveaux arrivants et nouvelles arrivantes, breton, alsacien, catalan et occitan, « pidgin-english » du Cameroun. De ces portraits se dégagent des identités linguistiques complexes, multiples et mouvantes, qui se rejoignent toutefois sur plusieurs aspects.

Les articles se distinguent et se complètent également par leurs approches méthodologiques, même si l'on peut les associer au champ large de la sociolinguistique. En effet, tous les auteurs semblent bien souscrire, implicitement si ce n'est explicitement, au «dogme central», pour reprendre les termes de Labov, voulant que l'on ne puisse comprendre la langue des individus sans connaître la communauté à laquelle ces individus appartiennent (Labov 1966 : 5). Si l'on peut donc caractériser la sociolinguistique comme l'étude du langage en rapport à la société, il reste que les questions pertinentes à cette étude et les méthodes nécessaires pour y répondre varient considérablement d'une école à l'autre. Labov a établi clairement dès 1966 les règles de ce qu'on appellera la sociolinguistique variationniste. Ces règles sont typiques des méthodes en sciences sociales : enquêtes rigoureuses, corpus représentatif, minimisation du rôle de l'enquêteur, analyses quantitatives basées sur des mesures statistiques éprouvées, objectivité des résultats. Cette approche quantitative, qui analyse des propriétés précises de la langue (variables linguistiques) en fonction de propriétés précises des locuteurs et locutrices (variables sociales), est largement répandue au Canada comme aux États-Unis. C'est celle qui est utilisée par Bigot et par Stelling et, dans une version hybride plus qualitative, par Mitchell et Ursulin Mopsus.

Plusieurs écoles de sociolinguistique privilégient plutôt des approches qualitatives et interprétatives de la langue, centrées plus sur les individus parlants, les situations d'interactions ou les valeurs sociales rattachées aux productions linguistiques. Ces approches sont plus prisées en Europe, au Royaume-Uni notamment et en France (voir Gadet 2003, ainsi que les travaux de Louis-Jean Calvet ou Claude Hagège pour une idée de la sociolinguistique hexagonale). Ces approches balaient un champ plus large (mais peut-être de façon moins ciblée) en laissant les corpus révéler les données intéressantes, corpus qui regroupent souvent des productions linguistiques de sources diverses,

\footnotetext{
3 J'utilise ici le terme "langue» de façon neutre, athéorique, en ignorant la distinction traditionnelle entre langues, dialectes, sociolectes, lectes, etc. Le terme désigne donc toutes les variétés linguistiques à l'étude, que ce soit des langues reconnues comme telles (l'hébreu, par exemple) ou des variétés d'une langue reconnue (le français québécois, par exemple). Cette simplification terminologique permet d'éviter la tâche délicate de distinguer entre langue et dialecte, tâche qui s'appuie tantôt sur des critères linguistiques tantôt sur des motivations politiques. Elle permet également de mieux représenter la réalité linguistique des locutrices et locuteurs et de la façon dont ils s'y réfèrent ; il eût été maladroit de parler de « variétés linguistiques identitaires », « de perceptions du dialecte » ou de « fierté du lecte ».
} 
comme c'est le cas ici : interviews semi-dirigées, conversations libres, articles de journaux, blogues, textos et graffitis. Dans ce numéro, plusieurs approches qualitatives sont utilisées, que l'on peut qualifier d'analyse du discours ou d'approche critique du discours (Blommaert 2005, 2010). L'analyse textuelle est au cœur de la démarche des deux auteurs qui adoptent une perspective historique, Berkaï et Moliner. Quant aux articles portant sur les attitudes linguistiques, ils ont recours soit à la méthode de «mesure directe », soit à la méthode du «traitement sociétal » aussi appelée «analyse de contenu ». Avec la première, retenue par Mitchell et Ursulin Mopsus, les locutrices et locuteurs répondent à des questions plus ou moins directement reliées à leurs jugements vis-à-vis de la langue. Avec la seconde, les analyses portent sur des textes du domaine public, qu'il s'agisse de textes officiels comme les bulletins du Ministère de l'Éducation nationale en France étudiés par Hamez, les chansons rapportées par Rostand, ou les forums de discussion analysés par Hallion.

Comme c'est le cas dans les autres branches de la linguistique et fort probablement dans toutes les sciences, un certain dogmatisme colore la recherche en sociolinguistique. Certains variationnistes sont peu friands de l'analyse du discours et certains analystes du discours ont le variationnisme en piètre estime. Le dogmatisme a ceci de bon qu’il assure une connaissance partagée parmi ceux et celles qui participent à la grande conversation scientifique, susceptible de faire avancer plus rapidement la science. Je dirai que le dogmatisme a ceci de moins bon que c'est une forme de prescriptivisme, l'imposition d'une norme unique dans une communauté donnée. Bien sûr, une norme partagée est nécessaire dans une communauté scientifique comme dans une communauté linguistique pour assurer l'intelligibilité, mais lorsque cette norme ne devient que prescription et proscription, elle risque de museler les identités locales, marginales, différentes, qu'elles soient linguistiques ou scientifiques. Heureusement, il existe des lieux de rencontre permettant des échanges non partisans et c'est précisément ce que propose ce numéro : un lieu de rencontre où les recherches sur le prescriptivisme et le patriotisme peuvent s'éclairer les unes les autres, à l'abri du dogme, et éclairer les uns et les autres, qu'ils soient linguistes, littéraires ou didacticiens.

Pour faire bon accueil à une diversité de lectrices et lecteurs intéressés par les études françaises, la suite de cette présentation situe les contributions à ce numéro dans une perspective plus large, faisant ressortir les singularités, les points de convergence et les aspects complémentaires, pour chacune des trois parties. La prochaine section apporte d'abord des précisions sur les concepts mêmes qui ont inspiré les articles de ce numéro : le prescriptivisme et le patriotisme. 


\section{Prescriptivisme et patriotisme : concepts et relations}

Précisons d'entrée de jeu que les termes de prescriptivisme et de patriotisme sont pris ici dans une acception plus large que ce que la tradition ou le sens commun comprennent généralement. Pour le prescriptivisme, il n'est pas seulement question de dictionnaires et de grammaires, de l'Académie française et de l'Office québécois de la langue française, mais aussi de ce sens instinctif de la prescription que possèdent, à des degrés divers, tous les locuteurs et locutrices d'une langue donnée. Pour le patriotisme, il n'est pas seulement question de fierté et de revendications linguistiques, de loi Toubon (France) et de loi 101 (Québec), mais aussi de ce patriotisme tranquille que l'on appelle généralement l’identité.

\section{- Le prescriptivisme}

Par définition, le prescriptivisme linguistique prescrit et proscrit: il impose une vision normative d'une langue donnée en stipulant quelles en sont les propriétés acceptées (et donc acceptables) et quelles en sont les propriétés prohibées. Cette vision implique que les traits linguistiques (prononciation, item de vocabulaire, tournure syntaxique) sont soumis à des jugements de valeur qui s'étendent également aux variétés linguistiques qui présentent ces traits. Si le prescriptivisme reconnaît l'existence de la variation linguistique, il ne reconnaît pas la légitimité de cette variation. Le prescriptivisme constitue somme toute une forme de purisme délégué, qui impose à autrui les objectifs de préservation de la pureté de la langue qu'il s'impose à lui-même.

Par définition également, le prescriptivisme est intimement lié à la notion de norme. Et c'est là que les choses se compliquent, la norme correspondant à au moins deux réalités imbriquées : norme d'usage et norme de bon usage (Vézina 2009) ou norme et surnorme (Garmadi 1981). La norme (d'usage) est simplement cet inventaire des traits linguistiques partagés par l'ensemble des locuteurs et locutrices d'une variété linguistique donnée, la façon de parler qui est en usage à une époque donnée et dans un lieu donné. Bref, tous les traits qui font qu’une langue est reconnue comme du français ou du français québécois ou du français québécois soutenu ou du français québécois populaire de Montréal, etc. Maîtriser une langue, c'est en connaître la norme (d’usage). Les grammaires descriptives - celles qui sont écrites par des linguistes plutôt que par des grammairiens ont pour objet de rendre explicite cette norme, sans porter d'autres jugements que des jugements de grammaticalité. 
La phrase Ca l'a l'air que mon chum va perdre sa job est acceptable, grammaticale; elle correspond à l'usage au Québec et s'avère donc conforme à la norme, ou du moins à une norme. En effet, elle présente des traits déviants par rapport aux standards du français de Paris, de Bruxelles ou d'Abidjan : «l'» épenthétique (ça l'a plutôt que ça a), anglicisme (chum), genre féminin de job. Avant même que l'on prenne en compte l'aspect surnormatif, le concept de norme se complique et ce, pour toutes les langues dont l'usage n'est pas circonscrit à un territoire restreint. C'est évidemment le cas pour le français, parlé comme langue maternelle de souche sur trois continents : parle-t-on alors de norme endogène (norme locale, développée au Québec, en Belgique ou en Côte d'Ivoire) ou de norme exogène (norme provenant d'une autre communauté, développée en France) ? Ou parle-t-on plutôt, d'une langue « pluricentrique » $\left(\right.$ Clyne 1992) ${ }^{4}$ ?

La phrase ci-dessus est donc grammaticale, mais peu acceptable au Québec dans un registre soutenu ou à l'écrit. Tout ce qui est acceptable est grammatical, mais l'inverse n'est pas vrai. Tout ce qui est grammatical n'est pas acceptable, puisqu'une forme grammaticale doit en plus passer le test de la norme du bon usage (surnorme) pour être jugée acceptable. La surnorme définit en effet «ce qui doit être choisi si on veut se conformer à l'idéal esthétique ou socioculturel d'un milieu détenant prestige et autorité » (Garmadi 1981: 123). Elle ne comporte que les traits linguistiques de la belle langue, de la langue correcte, utilisée par les groupes qui détiennent le pouvoir de servir de modèle à l'ensemble de la communauté. Bref, elle correspond au bon usage. La distinction entre norme et surnorme s'applique à toutes les langues qui ont des registres d'usage (autant dire toutes les langues) et à toutes les langues qui sont parlées dans des communautés socialement stratifiées (autant dire toutes les langues).

Le prescriptivisme est donc l'imposition de la surnorme comme modèle linguistique supérieur à tout autre et qui sert d'étalon pour mesurer la qualité des productions linguistiques (et par extension, la qualité des locuteurs et locutrices). La coercition avec laquelle le modèle est imposé varie considérablement d'une communauté à l'autre et peut prendre diverses formes: lois et règlements, programmes d'enseignement, médias, pression sociale, entraves à la mobilité sociale, etc. Le prix à payer pour ne pas se conformer à la surnorme varie également, mais il ne sera jamais nul. La surnorme est en effet la variété linguistique « légitime », au sens de Bourdieu, dont l'usage procure un capital symbolique et même matériel. Les situations d'échanges sociaux constituent en effet un marché linguistique dont les structures «s’imposent comme un système de sanctions et de censures spécifiques » (Bourdieu 1982 : 14).

\footnotetext{
${ }^{4}$ Je reviendrai sur le concept de pluricentrisme à la section 3.
} 
C’est peut-être parce que les conséquences néfastes des comportements non normatifs sont reconnues par les locuteurs et locutrices que le terme "prescriptivisme » n'est pas tout à fait neutre, moins certainement que ne l'est le terme «standardisation». Une certaine connotation négative le colore, l'associant aux iniquités sociales, à l'inégalité des chances, au pouvoir abusif des décideurs ou des élites sur les autres classes de la société. Le prescriptivisme est-il réellement un abus de pouvoir? Est-il un mal nécessaire ? Peut-il être un bienfait ? Nous reviendrons sur ces questions aux sections 3 et 5 .

\section{- Le patriotisme}

Au sens propre, le patriotisme est un sentiment d'appartenance à la patrie, voire un amour de la patrie qui motive les patriotes à la protéger. Dans ce recueil, le terme est pris au sens propre, mais avec un usage plus large du terme «patrie »: «la vraie patrie est celle où l'on rencontre le plus de gens qui vous ressemblent », écrivait Flaubert dans Rome, Naples et Florence (1826). Le patriotisme est donc ce sens d'un nous qui s'oppose à un eux, même si ce eux peut inclure le nous. Ce sentiment d'appartenance présuppose l'existence d'une certaine identité qui marque le groupe: identité ethnique, sociale, culturelle, nationale et, bien sûr, linguistique.

La construction de l'identité repose sur un paradoxe nécessaire. Le nous n’existe que de façon contrastive, en opposition au vous, au eux que sont les autres ; mais ces autres dérangent ou menacent précisément à cause de leur différence. Cela explique ce « jeu subtil de régulation qui s’instaure dans toutes nos sociétés (seraient-elles les plus primitives) entre acceptation ou rejet de l'autre, valorisation ou dévalorisation de l'autre, revendication de sa propre identité contre celle de l'autre » (Charaudeau 2009 : 16). L'identité est donc une réalité mouvante, qui doit constamment s'adapter et se réadapter en fonction de l'identité du groupe contre lequel elle se définit. L'identité est aussi une réalité imbriquée, stratifiée, comme une poupée gigogne : l’identité d'un groupe est contenue dans celle d'un groupe plus grand, elle-même contenue dans une identité plus large, etc.

La langue n'est pas un matériau essentiel dans la construction identitaire ; plusieurs groupes bâtissent leur identité sur une base ethnique, nationale, religieuse ou même culturelle ${ }^{5}$ qui n'inclut pas la langue. Par ailleurs, pour les groupes qui la retiennent comme substance identitaire, la langue revêt

\footnotetext{
${ }^{5}$ Je dis «même culturelle » parce que, bien qu'elle soit très souvent symbiotique, la relation entre langue et culture n'est pas indispensable. C'est peut-être ce qui explique que dans la Convention sur la protection et la promotion de la diversité des expressions culturelles de l'UNESCO (2005), le terme "langue » n'apparaisse qu'une fois, pour préciser que les mesures de protection et de promotion de la diversité comprennent « les mesures relatives à la langue utilisée pour lesdits activités, biens et services ».
} 
souvent un caractère fondamental, central, vital. C'est le cas, par exemple, du français au Québec ou du flamand en Belgique (voir Remysen 2010), pour prendre deux exemples très connus.

La construction identitaire est, par définition, complexe «parce qu'elle résulte d'un croisement de regards : celui du sujet communiquant qui cherche à la construire et à l'imposer à son partenaire, le sujet interprétant, lequel ne peut s'empêcher, à son tour, d'attribuer une identité à celuici en fonction de ses propres a priori » (Charaudeau 2009 : 27-28). Lorsque la langue est un matériau essentiel de l'identité, les a priori vont s'y appliquer : ce sont les attitudes linguistiques. Elles portent à la fois sur la variété linguistique (le français populaire de Montréal, par exemple), les marqueurs linguistiques de cette variété (utilisation de char plutôt que voiture, diphtongues lourdes dans la prononciation de formes comme père ou fort, etc.) et les locuteurs et locutrices de cette variété. Les attitudes linguistiques sont ces états mentaux, déclenchés par un stimulus linguistique, qui interviennent entre un stimulus et sa réponse, et qui dictent le comportement résultant (Lambert 1967 ; Mackey 1976). Une attitude est positive ou négative, et se combine à d'autres attitudes positives ou négatives, pour former un ensemble dont l'homogénéité est toute relative. Quel que soit leur groupe d'appartenance, et quel que soit le rôle que joue la langue dans le sentiment d'appartenance, les sujets parlants ont donc des attitudes linguistiques portant sur leur propre groupe comme sur tous les groupes avec lesquels ils interagissent. La langue est perçue comme un attribut du groupe, ce qui suscite nécessairement des stéréotypes et des jugements de valeur portant sur les locuteurs et locutrices de cette langue.

Les attitudes positives sont en quelque sorte la version internalisée, par le sujet parlant, du prestige de la langue. Ce n'est pas d'hier que le prestige est reconnu comme un attribut incontournable de la langue. Meillet notait déjà :

« ce qui fixe les formes et détermine le développement, ce sont les conditions sociales où se trouvent les sujets parlants. [...] toutes les langues connues, populaires ou savantes, trahissent la préoccupation d'un mieux dire qui partout a conduit les sujets parlants à emprunter le langage de ceux qui sont censés parler mieux. » (1926: 129)

Le prestige nous ramène évidemment aux notions de surnorme, de bon usage, de langue légitime, de capital symbolique, qui caractérisent la langue « de ceux qui sont censés parler mieux ». Le prestige nous ramène donc au prescriptivisme, mais reste en même temps un concept directement lié au patriotisme lorsqu'on prend en compte le prestige latent. Introduite par Labov (1966) et développée notamment par Trudgill (1972), la notion de « covert prestige » visait à expliquer pourquoi certains sujets d'une communauté linguistique étaient plus enclins que d'autres (les hommes plus que les femmes, particulièrement) à utiliser des formes non prestigieuses. Le prestige latent (ou prestige 
apparent ou norme implicite) confère à une variété linguistique un prestige différent du prestige légitime en lui prêtant des valeurs différentes de celles associées à la variété légitime. Ces valeurs plus intimes (douceur, empathie, humour, compassion) deviennent des symboles de solidarité au groupe, qui contrastent avec les valeurs d'autorité (clarté, élégance, pureté, compétence) qui caractérisent le prestige manifeste ou légitime.

En définitive, le patriotisme a besoin d'un prescriptivisme contre lequel s'identifier et le prescriptivisme a besoin des patriotismes (au pluriel) pour se donner un domaine d'application. Voyons brièvement les relations qui les unissent.

\section{- Prescriptivisme et patriotisme : des relations de frères ennemis}

Dans l'univers linguistique, il est difficile de caractériser les relations entre prescriptivisme et patriotisme de façon globale sans en dresser un portrait réducteur. Pour faire image, disons que c'est la relation entre deux frères ennemis. Ennemis, parce que le prescriptivisme sert d'outil de propagande au profit d'un projet plus large, unificateur (ou voulu comme tel), visant à niveler les variétés linguistiques dans un territoire donné et donc, à réprimer des patriotismes de façon plus ou moins directe, plus ou moins ouverte, plus ou moins coercitive. Frères, parce qu'un lien du sang unit le patriotisme et le prescriptivisme. Ils puisent tous deux à la même substance, qui mêle prestige, jugements de valeur, comparaison et rapport à l'autre. L'un comme l'autre n'existent qu'en rapport à un groupe, bien que ce ne soit pas toujours le même groupe.

La meilleure façon de cerner les relations qui unissent prescriptivisme et patriotisme est de les observer sur le terrain. C'est ce qu'ont fait les auteurs qui ont livré les huit articles de ce numéro. J'en présente la synthèse dans les trois sections suivantes.

\section{Le sujet parlant devant la prescription : perception des normes et des usages au Canada français.}

La norme : comment est-elle internalisée par le sujet parlant ? comment est-elle jugée par lui? Et le sujet parlant : comment est-il intégré à la norme ? comment est-il jugé par elle ? On observe souvent un décalage entre l'usage linguistique réel et la perception de l'usage, entre la réalité et une projection de la réalité, projection basée sur des observations impressionnistes, filtrées par les valeurs attribuées aux manifestations linguistiques. Cela s'explique par la dimension symbolique de la langue : ce qu'elle représente, ce qu’on souhaite qu'elle soit, indépendamment de ce qu'elle est. 
J'illustrerai cet aspect symbolique par l'exemple du Québec, en me référant particulièrement à l'ouvrage de Chantal Bouchard, La langue et le nombril: Histoire d'une obsession québécoise. Obsession, le terme n'est pas trop fort. Combien de livres, combien d'articles, d'émissions de radio et de télévision, de débats, de colloques, de discours et de «chicanes»a-t-on consacré au Québec à la langue? Et je ne parle pas ici des études savantes et des travaux de linguistes. Quelle Québécoise, quel Québécois n’a pas quelque chose à dire sur la question de la langue? Cette question, cent fois remise sur le métier, prend d'assaut la place publique de façon cyclique. Elle prend aussi d'assaut la place privée. En somme, elle prend toute la place, parce que «l'état de la langue est l'étalon par lequel nous mesurons notre évolution, et il en est ainsi parce que la langue est devenue l'élément central de notre identité » (Bouchard 1988 : 8). Parler de la langue, c'est parler de soi, de la société, de la société par rapport à soi, de soi par rapport à la société. D’où le décalage, puisque l'on n’a pas toujours une image juste de soi (ou de la société).

L'Histoire d'une obsession québécoise, depuis au moins les deux cents dernières années, est en fait celle d'un triumvirat : les conditions socio-économiques et politiques d'un côté, l'identité de l'autre, et enfin l'image de la langue. Que l'un des triumvirs attrape le rhume et les deux autres se mouchent. Ainsi, les plus grandes angoisses linguistiques apparaissent aux époques où les Canadiens françaisQuébécois $^{6}$ se sentent en perte de pouvoir politique ou économique. Les moments où le moral linguistique est au plus bas coïncident avec ceux où l'identité nationale est ébranlée dans ses composantes non linguistiques. La honte de la langue devient on ne peut plus explicite autour de 1950, époque où fusent les lamentations et les accusations portant sur cette bouillie informe, ce jargon infâme, ce joual sorti des bouches molles des locutrices et locuteurs canadiens-français. Encore une fois, le triumvirat agit de connivence. Cette vision la plus noire de la langue coïncide avec la grande noirceur politique et sociale de l'époque et avec la crise identitaire la plus aiguë que le Canada français ait connue. En effet, dans les grands bouleversements de l'après-guerre, il devient de plus en plus difficile de s’identifier aux bonnes valeurs de la ruralité, du catholicisme, des familles de douze enfants, qui définissaient traditionnellement l'identité canadienne-française. L'insécurité linguistique sert ici de symbole, de médium pour exprimer une insécurité plus profonde, identitaire et sociale. Tout observateur objectif de la langue aurait été à même de juger que la réalité n'était pas aussi sombre que les perceptions ne le laissaient croire.

\footnotetext{
${ }^{6}$ Le terme Québécois désignait au départ les habitants de la «Belle Province » de Québec. Il n’a commencé à remplacer le terme Canadien français qu’à partir des années 1960, prenant ainsi un sens ethnolinguistique, culturel et politique.
} 
Le Canada français-Québec est passé par une histoire qui se répète : les crises identitaires succèdent aux réaffirmations de soi, qui succèdent aux angoisses linguistiques. Et l'obsession est toujours là. Les vieux leitmotivs qui ont marqué tous les discours, à toutes les époques, reviennent encore chatouiller nos oreilles dans les ouvrages des professionnels de la langue, des éducatrices, des journalistes, des militants, des animatrices de télé, des puristes, des politiciens ou de Mme Tout-leMonde. On panique encore, on se lamente encore sur la qualité de la langue, le danger des anglicismes, les droits et devoirs de la littérature nationale, le sentiment d'amour-haine envers le français hexagonal, les difficultés extrêmes que pose la langue écrite. Les mêmes arguments, les mêmes débats resurgissent périodiquement, jamais réglés, parce qu'ils opposent des perceptions à des réalités.

Deux articles abordent ce décalage entre perception et usage. Le premier (Bigot) mesure les soi-disant écarts par rapport au français de référence dans le discours oral des élites québécoises. Le second (Hallion) dégage les représentations épilinguistiques du discours oral et écrit de la francophonie manitobaine.

\section{- Bigot : l'usage normatif à l'oral au Québec}

La norme linguistique est un éternel sujet de débat au Québec, qui oppose encore les linguistes, particulièrement en ce qui concerne la norme grammaticale orale. Davy Bigot apporte sa contribution au débat par une démonstration empirique: la réalisation réelle, dans une série d'entrevues télévisuelles (formelles), des marques de l'oral considérées typiques de l'usage québécois. L’objectif est de comparer la norme québécoise avec la norme du français de référence, tel qu'on la trouve décrite dans Le bon usage de Grevisse. S’il reconnaît que le Grevisse n'est pas à proprement parler une grammaire de l'oral, Bigot soutient qu'il s'agit là du «modèle grammatical de référence pour une grande partie de la francophonie ».

Bigot commence par rappeler les grandes lignes du débat opposant les défenseurs d'une norme internationale aux partisans d'une norme reflétant les usages réels au Québec. Des deux côtés, les positions sont basées sur des observations des usages linguistiques qui, tout en étant justes, ne sont pas toujours mesurées ou quantifiées. Dans le premier camp, on soutient que «les plus grands écarts entre le français québécois et le français standard se situent dans les registres familiers et populaires », qu'il n’y a pas de norme proprement québécoise et que «le seul français qui peut être enseigné est un français national, de niveau international». Dans le second camp, on évoque le 
consensus concernant l'existence d’un français québécois standard, cette «variété de français socialement valorisée » utilisée dans les situations de communication formelles.

L’usage québécois est analysé en fonction de 14 variables linguistiques, portant sur la morphologie et la syntaxe, qui sont mises en corrélation avec les variables sociales d'âge, de sexe et de catégorie d'emploi. Toutes variables confondues, l'usage des formes standard est très élevé et donc pas très distinct $\mathrm{du}$ «bon usage ». En rapport aux variables sociales, les résultats généraux indiquent que l'usage des formes standard est directement corrélé avec l'âge, les plus jeunes utilisant le moins les formes standard (63\% contre $80 \%$ pour les plus âgés). Cela suggère que « la norme grammaticale du français québécois tendrait donc petit à petit vers une utilisation plus prononcée de variantes populaires ». Par ailleurs, les femmes utilisent plus les formes standard (87\%) que les hommes $(78 \%$ ), ce qui confirme encore une fois la tendance maintes fois observée, depuis Labov (1966), qu'ont les femmes à favoriser les usages standard.

En somme, dans la pratique réelle, les élites sociales et culturelles ont recours à une grammaire de l'oral qui se démarque très peu de la norme préconisée par le bon usage. Pour conclure, Bigot offre une réponse claire à la question «Quel français doit-on enseigner au Québec? », question formulée au départ par l'Association québécoise des professeurs de français, puis reprise dans de nombreuses tribunes : «ce sont les formes prescrites dans des grammaires telles que Le bon usage de Grevisse-Goosse qui doivent être enseignées, puisque celles-ci font partie des tournures normatives employées par les élites québécoises dans un registre soutenu ».

S'agit-il là d'un décalage entre usage et perception de l'usage ? Cette norme québécoise seraitelle une projection de la réalité ? Pas tout à fait. Pour la syntaxe, il est vrai que l'usage québécois standard ne se démarque pas de la norme de référence, ou si peu. C’est la phonologie, la prononciation, l'accent qui rendent le français québécois, même très soutenu, reconnaissable. Et c'est aussi le vocabulaire, ces «milliers de mots et de sens propres à l'univers culturel, socioéconomique, géographique, etc. du Québec, et ce, dans tous les domaines de la vie courante» (Cajolet-Laganière et Martel 1996 : 2).

\section{- Hallion : représentations du français au Manitoba}

Au Manitoba, le français est une langue minoritaire, tant au sens démographique (parlée par $4 \%$ de la population) qu'au sens social (dominée par l'anglais), constituant ainsi un terrain propice à ces attitudes linguistiques contradictoires associées au prestige légitime et au prestige latent. Sandrine Hallion met au jour ces attitudes en analysant les représentations épilinguistiques, « ces conceptions 
ordinaires que se font les locuteurs des langues qu'ils parlent». Les données sont puisées à trois types de sources: orales (interviews semi-dirigées), écrites (introductions de glossaires, articles scientifiques et journaux) et, entre les deux, témoignages de jeunes sur un forum de discussion.

Outre l'attitude paradoxale attendue par rapport au vernaculaire, l'analyse de Hallion révèle que les discours épilinguistiques sont caractérisés par quatre grands thèmes récurrents. Le premier thème est la dévalorisation des variétés prestigieuses que sont le français québécois (soutenu), canadien (plus précisément, "radio-canadien»), hexagonal et international. En effet, les récentes vagues d'immigration francophone au Manitoba proviennent d'Europe et d'Afrique, et non plus du Québec, modifiant ainsi le tissu de la francophonie. Les discours valorisent ces diverses variétés prestigieuses, mais les critiquent aussi, dès qu'elles sont perçues comme dépréciant la langue vernaculaire, portant «atteinte au lien affectif et identitaire» qui lui est rattaché. On évoque la préciosité de ces variétés, ressenties comme non authentiques, et leur inadéquation au contexte local lorsque, par exemple, Manitoba est prononcé avec un «a» final identique à celui de tache ${ }^{7}$. On condamne également «l'anglomanie française » qui préfère le week-end, le parking et autres smokings aux créations lexicales francophones du Québec. Le deuxième thème est la recherche de l'intercompréhension, du mot juste et de l'équilibre : on loue «le parler des locuteurs qui réussissent à trouver un savant dosage dans le choix des mots et des expressions » et qui cherchent à se faire aisément comprendre.

Le troisième thème concerne la dévalorisation des variétés anglicisées conjuguée à la valorisation des archaïsmes. Comme le note Hallion, le mariage entre proscription des anglicismes et préservation des archaïsmes est une tendance qui caractérise depuis longtemps la mission prescriptive portant sur le français canadien. Ce n'est pas d'hier que l'on enjoint de «corriger nos anglicismes ». Au Manitoba, où le contact avec l'anglais est quotidien, l'anglicisation du français est une réalité reconnue par la communauté francophone, qui condamne l'usage de ce «franglais » qui n’est plus du français. On l'identifie d'ailleurs aux populations rurales, moins scolarisées, et à la « paresse intellectuelle », révélant ainsi la stigmatisation qui est associée à cette variété linguistique. Paradoxalement, le quatrième thème est la revendication des variétés anglicisées. Alors que certains réprouvent le mélange des codes, d'autres en reconnaissent le caractère inéluctable et quelques-uns, enfin, le revendiquent comme un moyen de préserver le français. C’est en fait un rejet d’une norme

\footnotetext{
${ }^{7}$ En français manitobain, comme en français québécois, un «a » en position finale de mot est postérieur (comme dans pâte) et non pas antérieur (comme dans patte). Les témoignages recueillis par Hallion montrent bien que les locuteurs et locutrices ont conscience des règles phonologiques qui caractérisent leur variété linguistique, le français du « Manitobâ ».
} 
trop puriste que cette position révèle et le rejet des attitudes négatives portées sur leur franglais - et donc sur leur identité - que les locuteurs et locutrices expriment ici.

Pour conclure, Hallion nous rappelle l'importance des attitudes linguistiques en situation minoritaire. Pour certains membres de la communauté franco-manitobaine, le franglais est le seul répertoire francophone disponible, en plus, bien sûr, de l'anglais. Si cette variété n'est pas reconnue comme légitime, si elle est stigmatisée, au sein même de la communauté qui l'abrite, il y a risque d'enlever à ceux et celles qui l'utilisent toute aspiration à la préserver, les privant ainsi « de leur capacité d'expression en les faisant se taire $[\ldots]$ en français ».

Cette stigmatisation des variétés anglicisées de français caractérise à des degrés divers toutes les francophonies canadiennes. Elle a marqué pendant longtemps le français populaire de Montréal, identifié, lui aussi, aux populations peu scolarisées, peu favorisées économiquement, peu cultivées des quartiers populaires. Les revendications culturelles liées à cette variété de français montréalais, à ce «joual», comme on l'a nommé dans les années 1960, ont adouci depuis la stigmatisation. Toutefois, la chasse aux anglicismes se poursuit toujours.

\section{- Normes au pluriel ou norme plurielle}

Les deux communautés francophones canadiennes que nous venons de voir ont ceci de commun qu'elles se positionnent en-dehors du centre normatif qu'est la France : ce sont des communautés périphériques. À ce titre, elles se rejoignent sur quatre aspects : « $1^{\circ}$ une prise de conscience des différences linguistiques par rapport au centre ; $2^{\circ}$ une ambivalence à l'endroit des pratiques langagières du centre ; $3^{\circ}$ un sentiment d'insécurité linguistique plus ou moins fort et $4^{\circ}$ une volonté d'intervenir sur la langue » (Willemyns et Bister 1989, cité dans Remysen 2010).

Pour des langues transnationales comme le français, toutefois, il n'est pas clair qu'il n'y ait qu'un centre autour duquel gravitent toutes les périphéries. Bref, il semble bien que si toutes les périphéries sont égales, certaines sont plus égales que d'autres. Certaines communautés périphériques ont émergé comme de nouveaux centres normatifs, faisant ainsi de la langue une langue pluricentrique (Clyne 1992). Ces divers centres normatifs reconnaissent leur variété linguistique comme légitimement distincte, utilisée comme véhicule de l’identité, de la culture, d’une certaine vision du monde. Et :

«s'il est vrai que la vision pluricentrique postule l'existence de plusieurs centres normatifs à l'intérieur d'une communauté linguistique, elle ne nie pas qu'il existe des inégalités en termes de prestige sur le marché des échanges linguistiques [...] certaines communautés ont en effet un poids plus important que d'autres, ce qui rend leur variété plus prestigieuse, voire dominante. » (Remysen 2010) 
Le français n'est donc pas une seule planète autour de laquelle gravitent des satellites, mais bien un système solaire incluant deux, trois, quatre planètes dotées chacune de leurs satellites. Le Québec est certainement l'une de ces planètes, autour desquels gravitent les variétés de français canadien horsQuébec et de français de Nouvelle-Angleterre.

Les communautés canadiennes ne sont pas les seules à présenter ces rapports enchevêtrés entre les normes qui caractérisent les diverses périphéries ou les divers centres de langues transnationales. Dans la plupart des communautés qui composent la francophonie (voir Moreau 1999), les locuteurs et locutrices ont bien internalisé ces inégalités dans le prestige attribué aux variétés linguistiques. Ils ont adopté l'idée que le français hexagonal est le seul bon français, tout en conservant un attachement à leur variété locale :

«dans le même temps qu'ils considèrent l'usage de France (conçu au singulier) comme équivalant à la norme, les francophones de la périphérie associent aussi des valeurs négatives à cette variété normée, lorsqu'elle est pratiquée par un des leurs, qu'ils accusent, en Belgique, de "fransquillonner", en Suisse de "raffiner", au Québec de "parler pointu" ou de "parler avec la gueule en cul de poule", au Sénégal de "faire le malin", d'être un "doseur" ou une “ciip-ciip", de renier leurs racines ». (Moreau 1999, non paginé).

La francophonie témoigne donc à grande échelle de cette ambivalence devant une double contrainte: d'une part, parler le bon français qu'est le français hexagonal et, d'autre part, ne pas parler comme les Français et Françaises, cette variété trop lointaine voire inaccessible (Moreau 1999).

Bien que la francophonie du Québec dispose d'un centre normatif local, lui permettant de prendre plus aisément ses distances par rapport au centre normatif ultime qu'est le français de France, elle n'est pas entièrement protégée de cette ambivalence. C'est ce qui explique l'hostilité générale qui s'est manifestée à chaque publication d'un ouvrage de référence proprement québécois, tels le Dictionnaire du français plus (1988), le Dictionnaire québécois d'aujourd'bui (1992, de la famille Robert) ou, plus récemment, le Dictionnaire bistorique du français québécois (1998).

La francophonie au Manitoba se présente comme un exemple particulièrement intéressant pour ce qui est des relations complexes entre les diverses normes d'une langue pluricentrique. Le franco-manitobain de souche est dans une relation enchâssée par rapport à la norme locale, le français du Québec dont il est d'ailleurs un rejeton. Il est aussi dans une relation enchevêtrée avec la norme de l'autre centre, la France, telle qu'elle se manifeste directement chez les locutrices et locuteurs nouvellement arrivés d'Europe et indirectement chez ceux et celles qui proviennent d'Afrique. Hallion parle d'ailleurs de «variétés prestigieuses » au pluriel. 
Avec une puissance démographique aussi faible (4\% de la population de la province), avec des attitudes linguistiques globalement négatives, en l'absence d'une norme linguistique proprement endogène (la norme locale provenant du Québec), avec toutefois un certain support institutionnel (médias, culture, école), la communauté franco-manitobaine présente un rare degré de résistance à l'assimilation linguistique ou, pour reprendre le terme qui fait l'objet de la section suivante, un degré étonnant de vitalité linguistique.

\section{Résistance passive, résistance active : vitalité linguistique et revitalisation}

La mondialisation ou, si on me permet ce terme à la mode, la globalisation, représente probablement l'exemple suprême du prescriptivisme. Les accords internationaux abattant les barrières tarifaires et ouvrant les frontières, les mouvements massifs de population, les moyens de communication hautement technologiques, Hollywood, l'internet, etc., sont autant de mécanismes ayant permis une standardisation de la vie quotidienne. Sauf pour ce qui est des plus démunis (et malheureusement ils sont légion), les biens de consommation, les modes de vie et même les idées sont partagés à l'échelle planétaire. Caractérisée par l'urbanisation et l'industrialisation, la surconsommation et la surexploitation des ressources naturelles, la mondialisation menace la diversité des espèces naturelles, mais aussi la diversité culturelle et linguistique.

Les scénarios les plus optimistes prévoient que la moitié des langues existantes seront éteintes d'ici la fin du siècle (Krauss 1991; Hagège 2000). Parmi les quelque 6000 langues du monde ${ }^{8}$, environ la moitié est parlée par moins de 3000 locuteurs et locutrices, donc par de petites populations, dont la singularité culturelle est souvent liée à une relative autonomie ou à un certain isolement. Que la mondialisation rejoigne ces communautés et la singularité aura du mal à se défendre. Au Canada seulement, plus d'une vingtaine de langues autochtones (abénaki, cayuga, haisla, kutenai, sagish, etc.) sont éteintes, presque éteintes ou sérieusement menacées.

Les facteurs permettant de prédire quel sera l'avenir d'une langue donnée sont difficiles à cerner : les mêmes facteurs favorisant le maintien de la langue dans une communauté donnée vont, dans une autre, entraîner son déclin (voir Dorian 1981, 1989 ; Jones 1998), comme nous le verrons plus loin (section 4). Un de ces facteurs se démarque toutefois en ce qu'il semble favoriser, dans un

\footnotetext{
${ }^{8}$ Le nombre total de langues varie d'un auteur à un autre (de 5000 à 7 000), en grande partie à cause des critères utilisés pour distinguer entre langues et dialectes.
} 
très grand nombre de cas, le maintien des langues minoritaires; c'est la diglossie. La diglossie (Ferguson 1959 ; Fishman 1967, 1972) est la coexistence au sein d'une communauté de deux variétés linguistiques, ayant un éventail de fonctions sociales complémentaires (domaines d'usage) et se plaçant dans une relation hiérarchique : l'une des variétés est haute (langue du pouvoir, du prestige, des situations formelles) alors que l'autre est basse (langue dominée, vernaculaire, des situations informelles). Selon Fishman (1967 : 36), le bilinguisme sans diglossie est une « situation transitoire » : sans un usage complémentaire des deux langues, sans une distinction claire entre les valeurs et les domaines d'usage associés aux deux langues, la langue dominante a tendance à déloger la langue dominée. Cette dernière, ne remplissant aucune fonction propre, est en quelque sorte jugée redondante et inutile, ce qui encourage le transfert («language shift») à la langue dominante, qui constitue le meilleur capital linguistique.

Quels que soient donc les facteurs en jeu, le processus d'érosion linguistique se présente comme une chaine d'événements impliquant quatre composantes, se succédant tout en se chevauchant. Le processus est d'abord enclenché par un changement majeur dans l'environnement, qui brise l'intégrité de la communauté linguistique et place la langue en situation minoritaire (colonisation, migration, déclin de la population, guerre et service militaire, unions exogames, urbanisation, industrialisation, etc.). Deuxièmement, les attitudes linguistiques deviennent plus négatives : le prestige de la langue diminue ; la langue est stigmatisée, parfois même au point où elle est perçue comme une entrave à la mobilité sociale, comme un handicap. Troisièmement, le comportement verbal des locuteurs et locutrices est atteint : la langue dominée perd des fonctions sociales (des domaines), la norme linguistique s'étiole, la diglossie se réduit et la transmission intergénérationnelle est interrompue. Enfin, la structure de la langue même est atteinte : elle est simplifiée et régularisée (réduction de la morphologie flexionnelle, remplacement du synthétique par l'analytique, abandon de catégories ou de distinctions, changements phonologiques, réduction de la variation stylistique).

Le processus d'érosion est plus ou moins rapide selon les situations et plus ou moins complet. Il n'atteint pas toujours la phase finale et peut même, soutenu par des activités d'aménagement linguistique, être renversé. L’aménagement linguistique est cette somme des efforts conscients, délibérés et soutenus faits pour changer la forme, la fonction ou le statut d'une langue dans une société. Il peut porter sur le corpus (normalisation, codification, modernisation du vocabulaire) comme sur le statut (élargissement des domaines d'usage et amélioration de la perception du statut) (Haugen 1972). 
Dans ce numéro, trois articles se penchent sur des situations linguistiques où la langue s'érode, où le transfert à une langue dominante est bien engagé, à des stades différents du processus. Dans les deux premiers textes (Mitchell et Ursulin Mopsus; Stelling), il s'agit de langues immigrantes (créole saint-lucien aux Îles Vierges, français en Nouvelle-Angleterre), donc minoritaires, mais bien implantées dans leur communauté. Dans le troisième (Berkai), il s'agit d'une langue qui avait dépassé le statut de langue moribonde pour atteindre celui de langue morte, l’hébreu.

\section{- Mitchell et Ursulin Mopsus : des attitudes linguistiques contradictoires}

À Sainte-Croix (Îles Vierges américaines), les Saint-Luciens constituent une part importante de la population, dépassant même le nombre de Cruciens de souche. Leur créole (qu'ils nomment «patois») est donc une langue minoritaire, qui se partage le marché linguistique avec l'anglais (américain et caribéen), l'espagnol et le créole crucien. Edward S. Mitchell et Diana Ursulin Mopsus analysent les usages de ces quatre langues et les attitudes qui s'y rattachent, en fonction de deux variables sociales : le sexe et le niveau d'éducation (pré-universitaire et universitaire) ${ }^{9}$. Leurs résultats montrent un ensemble paradoxal d'attitudes linguistiques, où la fierté côtoie le tabou.

Si la famille est le domaine par excellence pour l'usage du patois, celui-ci est également utilisé avec les amis et, dans une très faible mesure, au travail. Rares sont les participants ou participantes qui ont indiqué parler le patois avec tous les créolophones (12\%). L’usage du patois en public est donc très limité et, bien que $37 \%$ le jugent légitime, il est mal perçu par la majorité (62\%). Cela n'est pas parce que le patois n'est pas reconnu comme une « vraie » langue ; au contraire, $93 \%$ des sujets interviewés reconnaissent le patois comme une langue. Et cela n'est pas parce que les attitudes envers le patois sont globalement négatives. On trouve seulement trois mentions donnant le patois comme une langue bâtarde ou grossière contre 25 mentions le présentant comme une langue spéciale, unique, belle, bénéfique, dont on peut être fier, bref, la meilleure. Le tabou contre l'usage public du patois ne provient pas non plus d'un malaise par rapport à la langue. Seuls $25 \%$ des répondants et répondantes désignent l'anglais comme la langue avec laquelle ils sont les plus à l'aise ; dans les autres cas, la langue préférée est le patois, seul ou en combinaison avec l'anglais.

Selon Mitchell et Ursulin Mopsus, ce tabou contre l'usage public du patois trouve son origine dans les idéologies plus profondes associées aux langues en présence, explication qui se voit appuyée par les résultats qui prennent en compte le niveau d'éducation. En effet, le tabou est

${ }^{9}$ Dans cette présentation, je mettrai de côté les distinctions liées aux deux variables pour me concentrer sur les résultats généraux. 
exprimé par la majorité de ceux et celles qui ont un diplôme universitaire $(75 \%)$ alors que le droit de parler le patois en public est revendiqué à $67 \%$ dans le groupe moins scolarisé. Les locutrices et locuteurs plus scolarisés, ayant été plus exposés à l'anglais et plus en contact avec des noncréolophones, auraient développé une sensibilité plus grande au prestige de l'anglais et au stigma rattaché au patois comme marqueur social. D’ailleurs, les attitudes envers l'autre créole vont dans le même sens: c'est surtout chez le groupe le plus scolarisé que le créole crucien est perçu négativement.

Mitchell et Ursulin Mopsus envisagent l'avenir du patois à Sainte-Croix avec pessimisme, prédisant son abandon au profit de la langue de la «domination globale », l'anglais. Déjà, les enfants apprennent de moins en moins le créole. Les auteurs notent bien quelques facteurs favorisant le maintien de la langue : réseaux sociaux forts dans la communauté, contacts fréquents avec la famille à Sainte-Lucie, sentiment puissant de fierté nationale et d'identité ethnolinguistique. Ils notent cependant la présence de puissants facteurs adverses : la situation dominante de l'anglais, le prestige de l'anglais, l'absence du patois dans les médias, ainsi que les facteurs idéologiques, qui associent un statut supérieur aux langues standardisées (surtout européennes) et qui idéalisent le monolinguisme pour mieux le promouvoir.

Les auteurs ont probablement raison d'être pessimistes. Les créoles sont menacés en plus d'un endroit des Caraibes, et les créolophones transfèrent à la langue coloniale, que ce soit l'anglais, le français ou l'espagnol. Le prescriptivisme idéologique met une pression très forte sur des langues comme les créoles qui, il n'y a pas cinquante ans, étaient encore considérées comme des langues incomplètes. Même à Sainte-Lucie, où il est soutenu par quelques efforts d'aménagement linguistique (cours de créole aux enfants et aux adultes, dictionnaires), le patois perd des domaines d'usage, s'anglicise et commence à se simplifier (surtout en milieu urbain).

\section{- Stelling : une vitalité linguistique atypique}

Le français parlé aux États-Unis se porte mal, de la Nouvelle-Angleterre à la Louisiane. Louis E. Stelling nous présente un diagnostic de son état d'érosion à Southbridge (Massachusetts) et Woonsocket (Rhode Island). Ces deux communautés jadis majoritairement francophones (60\% de la population en 1900) ont vu l'usage du français perdre du terrain, abandonnant tour à tour des fonctions sociales : les groupes sociaux et les lieux de travail (les «facteries» qui avaient attiré les Canadiens français), puis l'école et l'église, pour enfin se réduire au seul domaine de la famille et encore, concurremment avec l'anglais. En perdant des domaines d'usage, la langue devrait s'être 
simplifiée, et Stelling mesure l'usage de l'auxiliaire avoir (plutôt que être) avec les verbes inaccusatifs comme aller, venir ou mourir pour évaluer le degré de cette simplification. Étonnamment, les résultats démontrent un taux global de l'usage d'avoir assez bas (34\%), qui se compare à celui que l'on retrouve dans certaines variétés plus robustes de français américain, le français québécois et le franco-ontarien; on est loin de la situation en Louisiane où l'usage d'avoir s'est généralisé.

Stelling nous montre que le degré de simplification dans l'usage de l'auxiliaire varie de façon significative en fonction de plusieurs facteurs extralinguistiques : la fréquence d'emploi du français (nul, rare, fréquent ou quotidien), l'âge, le sexe, le niveau générationnel (première, deuxième ou troisième génération en terre américaine), le statut socio-économique, la ou les langues de scolarisation (français, français langue seconde, bilingue). On s'attendrait à ce que l'usage non simplifié, être, se retrouve majoritairement chez les locutrices et locuteurs les moins touchés par le transfert à l'anglais, ceux et celles qui ont une meilleure maîtrise de la langue, soit : les plus âgés, de première génération, scolarisés en français, parlant français tous les jours. Pour ce dernier facteur, les résultats vont dans le sens attendu : l'usage de être est favorisé par la scolarisation en français (qui a eu lieu majoritairement au Québec) et, dans une moindre mesure, en français langue seconde. Pour les autres facteurs, les résultats étonnent, encore une fois. D’abord, c'est au contraire l'usage simplifié que l'emploi quotidien du français favorise. Ensuite, si les plus jeunes utilisent plus avoir (56\%) que les plus âgés (25\%), comme on s’y attendait, il n’y a pas de différence notoire parmi les plus jeunes, c'est-à-dire les moins de 70 ans! C'est donc dire que ce changement vers la simplification n'a pas progressé avec le temps. Enfin, si la première génération est bien celle qui utilise le moins avoir, c'est la deuxième génération qui l'utilise le plus, et non pas la troisième (la plus jeune, normalement la plus assimilée à la langue dominante).

Stelling attribue cette surprenante vitalité du français au fait que les deux communautés ne représentent pas des cas typiques de minorisation linguistique : le français est par ailleurs une grande langue internationale, et les locutrices et locuteurs ont presque tous été exposés à d'autres variétés de français, à l'école, dans les médias et dans leurs déplacements à l'extérieur de la communauté. Ils ont donc en fait été exposés à une norme non seulement prescriptive, mais aussi proscriptive, stigmatisant les usages fautifs tels que l'usage de l'auxiliaire avoir avec certains verbes. Stelling conclut que «la proscription linguistique peut ralentir voire bloquer certains développements linguistiques dans des situations de transfert linguistique » lorsque les facteurs sociolinguistiques nécessaires sont présents, tels que la scolarisation en langue maternelle et le conservatisme linguistique. 
La situation à Southbridge et Woonsocket présente en effet tous les ingrédients qui finissent généralement par attaquer l'intégrité de la langue : diglossie instable sinon inexistante, absence de fonctions sociales, absence de norme linguistique locale. Et pourtant la structure de la langue résiste, renforcée par le modèle normatif. La prescription joue donc ici un rôle positif, qui s'allie au patriotisme plutôt que de s'y confronter.

\section{- Berkaï : la revitalisation d'une langue morte}

Abdelaziz Berkaï nous présente une «leçon de patriotisme linguistique », la «success story » d'une revitalisation que l'on pourrait même qualifier de résurrection puisque l'hébreu, à la fin du $\mathrm{XIX}^{\mathrm{e}}$ siècle, n'était une langue vivante qu'à l'écrit. Pendant dix-sept siècles, et malgré quelques regains passagers, l'hébreu est resté une langue seconde limitée aux écrits littéraires et surtout religieux. Sous l’impulsion de Ben Yehuda, le «père de l'hébreu moderne », la langue allait redevenir une langue parlée (langue seconde) en l'espace d'une seule génération (1891-1916), pour devenir une langue maternelle à la génération suivante.

Pour Berkaï, deux facteurs se sont avérés cruciaux dans la résurrection de l'hébreu : les attitudes linguistiques et l'usage de la langue à l'école. En cette fin de XIX siècle qui voit la consolidation du nationalisme linguistique en Europe et l'émergence du mouvement sioniste, alors que les Juifs nouvellement arrivés en Palestine ottomane veulent se doter d'une lingua franca, deux candidats s'imposent, le yiddish et l'hébreu. En fonction de motifs purement utilitaires, soit d'établir rapidement une langue commune pour des fins de communication, le choix se serait porté tout naturellement sur le yiddish, langue partagée par la majorité des locuteurs et locutrices. Or ce sont les motifs patriotiques, identitaires, qui ont primé, ainsi que les perceptions associées aux deux langues. Autant les attitudes envers le yiddish étaient négatives (jargon, langue dégénérée, qui rappelle douloureusement l'exil et les persécutions), autant les attitudes envers l'hébreu étaient positives (langue plus que millénaire, chargée d'histoire, qui véhicule un héritage collectif). Le deuxième facteur, l'éducation, a rendu possible la généralisation et la diffusion de ce nouveau choix de code, qui s'est imposé lorsque la génération éduquée en hébreu a transmis ce nouvel héritage culturel à la génération suivante.

Cette résurrection est étonnante puisque, comme le note Berkaï, «les langues enseignées massivement sont généralement celles du pain. Or, il n’y avait rien à gagner matériellement à apprendre l'hébreu vers la fin du XIX siècle ». Pour Berkaï, l’avenir des langues « dépend en grande partie de la volonté de leurs locuteurs à les parler et à les adapter à leur temps ». Il est optimiste 
quant aux possibilités de survivance, et même d'expansion, des langues menacées et moribondes. « Si une conservation scripturaire rudimentaire et une transmission par l'enseignement traditionnel ont été les moyens ayant permis à l'hébreu de redevenir vivant», on peut effectivement entretenir des espoirs pour les langues qui disposent d'Internet et autres nouvelles technologies pour consigner plus facilement et transmettre plus efficacement un héritage linguistique.

En effet, l'une des grandes forces motrices derrière le choix d'un code donné dans une situation multilingue est précisément la valeur de ce code sur le marché linguistique, le capital symbolique qu'il représente, son efficacité à promouvoir la mobilité sociale. Mais encore une fois, comme on l'a vu précédemment avec le saint-lucien et le français franco-américain, l'identité semble agir comme une force plus grande encore, qui mène les membres d'une communauté à sacrifier des gains en capital ou en pouvoir contre une identité linguistique qui ne promet pas de gains bien palpables. Bref, tout ce qu'apporte cette identité, c'est une identité. C'est très peu et c'est aussi, semble-t-il, beaucoup; mais est-ce assez? J'aimerais partager cet optimisme quant à l'avenir des langues menacées, mais plusieurs raisons m’en empêchent, raisons qui sont exposées à la section suivante.

\section{- Un avenir incertain pour la diversité linguistique}

La première raison qui tempère mon optimisme est que, comme c'est le cas dans nombre de situations multilingues, les facteurs favorisant le maintien de la langue sont nombreux et complexes (comme on l'a vu plus haut). S'il est d'accord pour reconnaitre le rôle central des attitudes positives et de l'école dans la revitalisation de l'hébreu, Spolsky (2009 : 28-29) accorde un rôle majeur aux pionniers sionistes vivant en milieu rural, aux nombreux groupes activistes en Palestine ottomane (dont certains étaient soutenus de l'extérieur), à l'idéologie sioniste et même à la création des kibboutz, visant entre autres à procurer un environnement linguistique contrôlé favorisant (voir forçant) l'usage de l'hébreu. Il semble donc que plusieurs acteurs aient participé aux efforts d'aménagement linguistique et qu'il ne soit pas nécessairement si facile de reproduire les conditions gagnantes. Est-il possible de cerner celles qui ont assuré le succès de la renaissance de l’hébreu ? Plus généralement, comment prédire le résultat d'un processus déjà amorcé d'érosion linguistique? Comment en mesurer le stade d'avancement? Comment prédire le succès ou l'échec des efforts d'aménagement linguistique?

Cette question m'amène à la deuxième raison de mon pessimisme. Pour maintenir ou revitaliser une langue menacée, pour influencer son avenir, il nous faudrait un modèle qui permette 
de prédire le sort d'une langue donnée dans une situation donnée et donc d'identifier les activités d'aménagement linguistique qui seront couronnées de succès. Or, il ne semble pas y avoir de modèle qui possède ce pouvoir de prédiction. Giles et ses collaborateurs ont bien proposé un modèle qui mesure de façon très précise la vitalité linguistique d'une communauté donnée, mais ce modèle permet de dégager a posteriori une typologie des situations de maintien ou de perte, pas de prédire l'avenir d'une langue. Ce modèle (voir Giles, Bourhis et Taylor 1977) associe la vitalité linguistique à trois ensembles de facteurs: le statut (économique, social, sociohistorique et linguistique), la démographie (force numérique, distribution géographique, natalité, exogamie) et le support institutionnel (médias, législation, école, marché du travail, culture, religion). Le problème est que ces facteurs n'ont pas de valeur absolue ; certains peuvent aussi bien favoriser le maintien de la langue que sa perte; plusieurs sont presque toujours liés à d'autres facteurs dont ils deviennent, à toute fin pratique, indissociables.

Prenons l'exemple du français du Québec. Jusqu'à la Révolution tranquille (début des années 1960), le français avait une position peu enviable en termes de statut économique, social et même linguistique, les attitudes étant plus positives envers l'anglais et le français hexagonal. La langue jouissait en outre d'un support institutionnel limité (école, religion, médias). À partir des années 1970, un revirement de situation allait raviver la vitalité linguistique du français, avec l'essor économique du Québec, une ascension sociale des francophones, le développement d'attitudes positives envers le français québécois, la création d'outils d'aménagement linguistique (de corpus et de statut), les législations de protection de la langue et le rayonnement international de la culture québécoise (Céline Dion, le Cirque du Soleil, Robert Lepage). Tous ces facteurs sont dans une situation d'interdépendance, se soutenant l'un et l'autre, et il me semble difficile de déterminer leur part relative d'influence dans la revitalisation du français québécois. Cette difficulté est relevée par Spolsky tout au long de son ouvrage consacré à l'aménagement linguistique et le conduit même à se demander si les écosystèmes linguistiques ne sont pas des systèmes «chaotiques », aussi susceptibles de subir les surprises de «l'effet papillon » que les systèmes météorologiques (Spolsky 2009 : 260).

La troisième raison pour laquelle ma vision de l'avenir des langues n'est pas très optimiste est ce que j’appellerai la synergie négative. Lorsque le processus d'érosion est enclenché, certains facteurs qui le favorisent se renforcent mutuellement, avec un effet synergétique. Comme pour la situation du Québec rapportée plus haut, mais à l'envers. Le transfert à la langue dominante et les attitudes linguistiques négatives, par exemple, vont main dans la main : plus il y a de locuteurs et locutrices qui transfèrent, moins la langue a de valeur, et moins la langue a de valeur, plus il y a de 
locuteurs et locutrices qui transfèrent. Cette synergie négative mène le processus à un point de précipitation à partir duquel tout s'accélère. Cet effet de précipitation est bien visible lorsque l'on compare, sur plusieurs décennies, le taux d'assimilation à la langue dominante dans une population minoritaire. Pour le français cajun de la Louisiane, par exemple, si le taux d'assimilation par décennie ne dépassait pas les 3,8 \% jusqu'en 1950, il est passé à 38 \% pour la décennie 1950-1960 et à $58 \%$ pour 1960-1970 (Johnson 1976). Le problème, encore une fois, en est un de pouvoir prédictif : peuton anticiper le moment où sera atteint ce point de non retour et agir à temps pour le contrer ou au moins le repousser?

La dernière raison de mon pessimisme est la mondialisation, avec ses nouvelles réalités et ses nouvelles technologies qui, toutes mondiales qu'elles soient, n'impliquent pas tout le monde de la même façon. Les nouvelles technologies reposent sur l'écrit et la mondialisation risque en fait de creuser l'écart entre langues bien établies (codées, écrites, littéraires) et langues issues de la tradition orale. La revitalisation réussie de l'hébreu a bénéficié d'une tradition écrite millénaire et d'un héritage littéraire important. Le maintien du français en Nouvelle-Angleterre a profité du statut international du français et de la proscription véhiculée en grande partie pour l'écrit. Le patois de Sainte-Lucie, langue qui s'écrit depuis peu et dont la standardisation est à peine amorcée, se perd. La codification est-elle donc nécessaire au maintien d'une langue? L'expérience coloniale nous amène à répondre non. L'Afrique de l'Ouest, par exemple, colonisée par la France, la Belgique et l'Angleterre, a vu se perpétuer l'usage d'un grand nombre de langues et dialectes autochtones, bien avant que des efforts de codification écrite et d'alphabétisation des langues nationales ne soient faits. Mais est-ce encore possible aujourd'hui pour ces langues issues de la tradition orale ? Et pour combien de temps?

Globalement, la tendance va vers une dépréciation des traits distinctifs des communautés (mondialisation, concept de citoyen du monde) et vers un focus sur le pouvoir économique et politique de la langue plutôt que sur son pouvoir culturel et ethnique (Blommaert 2010). Et cela vient renforcer cette idée assez répandue selon laquelle le bilinguisme est "onéreux », voire dangereux, pour la communauté et même pour l'individu (Dorian 1988), soi-disant parce que l'acquisition et l'usage de la langue minoritaire peuvent mettre en péril la maitrise de la langue dominante. C'est le cas à Sainte-Croix ; c'est le cas des langues autochtones au Canada ; c'est le cas des langues des populations issues de l'immigration qui disparaissent souvent à la troisième génération ; c'est le cas des langues parlées par de petites communautés dans ces régions du monde qui présentent la plus grande diversité linguistique au kilomètre carré (îles du Pacifique, bassin amazonien) lorsqu'elles entrent en contact avec une langue bien établie. 
Un transfert à rebours est peut-être possible si des mesures concrètes et agressives sont prises pour regagner des domaines d'usage (et réinstaurer une diglossie stable), renforcer les compétences individuelles (en documentant, standardisant et enseignant la langue) et promouvoir le statut linguistique (et rendre les attitudes plus positives). Avec les moyens appropriés ${ }^{10}$, les efforts d'aménagement linguistique pourraient peut-être engendrer une revitalisation de la langue. Comme on l'a vu plus haut, le succès de cette revitalisation reste difficile à prédire, mais cela vaut certainement la peine d'essayer.

\section{Usages du pouvoir, usages militants : mêmes moyens, différents combats}

Trois articles illustrent les relations entre prescriptivisme et patriotisme, relations intestines, symbiotiques, parfois au point où les deux entités se confondent. Les deux premiers textes nous présentent le positionnement du pouvoir politique, étatique de la France vis-à-vis de variétés linguistiques marginales: les patois et autres langues régionales autour de la Seconde Guerre Mondiale (Moliner) et les langues des élèves nouvellement arrivés en France, depuis 1970. Le troisième article (Rostand) présente la situation compliquée du pidgin-english au Cameroun, qui coexiste avec deux grandes langues internationales, le français et l'anglais.

\section{- Moliner : réconcilier les patriotismes régionaux et l’idéal républicain}

Olivier Moliner nous présente une tranche de la longue histoire des débats portant sur la place des langues régionales en France, du point de vue particulier des parlementaires communistes, avant et après la Seconde Guerre mondiale. L'auteur vise à répondre à deux questions centrales : «Quels sont les argumentaires, les modèles politiques ou les réflexions auxquels les communistes se sont référés pour s'engager en faveur des langues régionales? Comment sont-ils parvenus à synchroniser patriotisme français, passé résistant, fidélité à la ligne du parti et engagement pour une diversité linguistique en France ?»

Comme nous le montre Moliner, la période précédant la Seconde Guerre (1925-1939) est marquée pour les communistes par « un dilemme des idéologies ». Ceux-ci sont tentés d'adopter une

\footnotetext{
${ }_{10}$ Malheureusement, ce ne sont pas toutes les communautés qui ont les moyens de prendre les mesures appropriées. Les langues en danger ou menacées d'extinction se trouvent souvent dans des nations en émergence qui ont des impératifs de développement plus urgents que l'aménagement linguistique (accès à l'eau potable et à la nourriture, soins médicaux, logement, éducation, etc.).
} 
conception lénino-stalinienne de la nationalité et des minorités nationales qui cadre mal avec l'idée française de la nation. En outre, ils doivent composer avec l'idéal « d'unité fraternelle » qui devrait unir les militants du parti, d'un côté, et les velléités séparatistes des communistes d'Alsace et de Lorraine, de l'autre. Dans le contexte de la montée du nazisme, les communistes n'ont d'autre choix que de se ranger derrière les intérêts nationaux, tout en soutenant les revendications linguistiques (mais pas nationales) de l'Alsace-Lorraine. La fin de cette période voit deux initiatives concrètes de reconnaissance des langues régionales : la loi Dahlet (1936), qui introduit le «bilinguisme intégral » français-allemand dans les institutions publiques des départements Bas-Rhin, Haut-Rhin et Moselle, et le rapport Desgranges (1937), qui vise à introduire «l'enseignement de la langue bretonne, parallèlement à celui de la langue française » mais ne sera pas mis en application.

Pendant la période de l'après-guerre (1945-1952), les communistes bretons sont à l'avantgarde des revendications linguistiques régionales. Ils réclament l'abrogation des règlements interdisant l'usage des langues régionales et une reconnaissance de statut pour le breton, bien sûr, mais aussi pour l'occitan. La loi Marty, votée en 1950, ajoutera le catalan aux cursus des deux universités de la région (Toulouse et Montpellier) et de certains autres établissements. À l’issue de la guerre, les revendications linguistiques régionales reviennent à l'ordre du jour, mais elles ne sont pas toutes logées à même enseigne; la présence de l'allemand en Alsace-Lorraine est un souvenir douloureux et gênant, qui contraste avec le passé résistant de la Basse-Bretagne ou de la Catalogne. Cela mènera à la loi Deixonne (1951), qui autorise l'enseignement facultatif des langues régionales de façon discriminatoire : elle s'applique au breton, à l'occitan, au basque et au catalan, mais exclut l'alsacien, le flamand et le corse.

Comme le montre Moliner en comparant les dossiers alsaciens, catalans et occitans, cette iniquité du traitement des langues régionales par la législation se reflète dans les prises de position communistes. Celles-ci trouvent leur fondement théorique dans la doctrine stalinienne, datant de 1913 et remise en circulation parmi les membres du Parti Communiste en 1945 : une minorité nationale ayant droit à une certaine reconnaissance doit se présenter comme une «communauté stable historiquement constituée, de la langue, de territoire, de vie économique, de formation psychique ». Or, cette doctrine a été interprétée de façon aussi floue qu'arbitraire, positionnant les langues régionales sur une hiérarchie: 1) les langues d'une «minorité linguistique/nationale » bilingue (alsacien et allemand); 2) les «langues familiales» des régions majoritairement francophones (breton et catalan); et 3) les langues dont l'histoire « est liée très tôt à celle de la France » et qui n'ont jamais eu de nationalité propre (l'occitan). En conclusion, Moliner rappelle le 
triple dilemme des communistes français qui devaient réconcilier «le patriotisme pour une République unie avec le français comme langue nationale, la conception marxiste-stalinienne et l'attachement aux langues régionales ». Ce dilemme n’a pas empêché les communistes de s'engager très tôt dans la défense des intérêts nationaux avec - et cela les distingue des autres partis - un appui collectif des groupes concernés.

Cette tranche d'histoire nous montre le patriotisme comme une réalité mouvante, que des événements politiques importants peuvent modifier radicalement et très rapidement. Le patriotisme des uns n'est pas le patriotisme des autres, même au sein d'une même communauté. Et ces patriotismes ne sont pas à l'abri de la prescription idéologique, de gauche comme de droite, dès qu'ils se frottent un peu aux sphères du pouvoir.

\section{- Hamez : le français tel que prescrit aux élèves nouvellement arrivés}

Depuis une quinzaine d'années, l'enseignement du français en France s'est donné une nouvelle perspective, un nouvel objet : le français langue de communication et de scolarisation ou, plus simplement, le français langue seconde. Marie-Pascale Hamez examine 40 ans de textes officiels, émanant surtout du Ministère de l'Éducation Nationale, qui préconisent les contenus et méthodes d'enseignement du français pour les élèves bénéficiant de dispositifs d'accueil particuliers. Son analyse s'inscrit dans deux grands axes : le statut accordé à la diversité culturelle et linguistique des élèves nouvellement arrivés et les construits théoriques, politiques et idéologiques (pas toujours explicites) qui sous-tendent les prescriptions.

Au début des années 1970, sont créés les premiers dispositifs d'accueil pour les élèves nouvellement arrivés en France. Alors que le monolinguisme caractérise toujours la didactique française, ce sont les méthodes de FLE (français langue étrangère) qui sont préconisées pour l'enseignement de la langue. Ces méthodes, où l'apprentissage de la production écrite n'est pas une préoccupation centrale, sont contestées puisqu'elles marginalisent «leur public d'élèves en les orientant vers les sections de l'enseignement spécialisé ». En 1975, on abandonne cette approche et on remet au goût du jour des dispositions qui prévalaient dans les années 1920-1930, soit «le maintien des enfants étrangers dans la connaissance de leur langue et de leur culture d'origine ». Ces nouvelles dispositions ont bien un fondement didactique (faciliter l'apprentissage du français en construisant sur les compétences de la langue maternelle), mais elles ont aussi, dans un contexte de crise économique, un fondement politique (préparer les élèves à leur éventuel retour au pays natal). 
En 1986, l'approche communicative en vogue inspire les nouvelles prescriptions qui encouragent, outre la langue orale, l'écriture nécessaire aux interactions sociales de tous les jours (les fameux documents «authentiques»), au risque de ne pas préparer adéquatement les élèves aux formes plus complexes qu'exigera plus tard la culture scolaire. C'est en 1995 qu'apparaissent pour la première fois les termes «français langue seconde» et «allophone» dans les textes officiels. Les programmes puisent alors aux théories en cours sur l'acquisition des langues secondes, sans trop préciser les moyens pour les appliquer en salle de classe. Ils préconisent un enseignement qui «considère les erreurs comme des témoignages d'un apprentissage en train de se faire, comme les manifestations d'un processus d'interlangue ». L'approche est développée et instrumentalisée en 2000, dans Le français langue seconde, qui offre des méthodes et des contenus spécifiques pour les classes officiellement reconnues comme plurilingues et pluriculturelles. On y voit réapparaître l'ancien modèle, abandonné depuis 15 ans, de la rédaction correspondant aux codes scolaires du cursus régulier. Pour compliquer les choses, les prescriptions de 2000 doivent être suivies tout en respectant une approche différente, préconisée par le Cadre européen commun de référence pour les langues, qui définit entre autres les contenus et épreuves menant à l'obtention du Diplôme d'Études de Langue Française (DELF), reconnu au niveau européen.

Hamez nous rappelle en conclusion les difficultés particulières liées à l'enseignement de la langue en classe d'accueil: hétérogénéité des classes d'élèves (âge, langue maternelle, niveau de scolarisation antérieur), manque d'outils pédagogiques et didactiques spécifiques au français langue de scolarisation, insuffisance des moyens accordés à la formation des enseignants et enseignantes. À cela, s'ajoute la difficulté particulière liée à la contradiction entre les deux paliers de prescription officielle: palier national préconisant d'enseigner un français langue de scolarisation et palier européen recommandant d'utiliser des outils conçus pour l'enseignement des langues vivantes étrangères.

Pour un État, l'éducation est un moyen par excellence d'influencer le comportement linguistique, voire identitaire, de ses citoyens et citoyennes. Les prescriptions institutionnelles en matière d'éducation agissent donc comme un révélateur des idéologies que l'État entretient en rapport à l'identité et à la langue, et en rapport à la façon dont les nouveaux citoyens et citoyennes sont intégrés (ou non) dans la société. Ce qu'elles révèlent pour la France est une situation répandue dans plusieurs communautés: l'existence d'un ensemble prescriptif à paliers multiples, où un standard local est contredit par un standard externe. Bien sûr, pour la France, il s'agit de standards 
éducatifs et non pas de standards linguistiques à proprement parler, puisque le français « international » est essentiellement perçu, en France comme ailleurs, comme le français hexagonal.

\section{- Rostand : du prestige latent à l'usage militant}

Ancienne colonie allemande, cédée ensuite à la France et au Royaume-Uni, le Cameroun indépendant se présente comme une mosaïque linguistique et culturelle: on y parle près de 200 langues autochtones, en plus du français et de l'anglais (langues officielles) et des deux langues issues du contact, le pidgin-english et le camfranglais. Pandji Kawe Guy Rostand nous propose un portrait de l'usage militant du pidgin-english dans les réclamations et les revendications identitaires camerounaises, attirant l'attention sur quelques leaders politiques et culturels qui ont reconnu le pouvoir mobilisateur de la langue.

Historiquement, la position de l'État avait toujours été de réprimer l'usage des langues locales, en tolérant tout juste le pidgin-english, nécessaire aux échanges commerciaux. Dans la foulée de l'indépendance et de la réunification des territoires français et britanniques, le pays doit se choisir une langue officielle. Le pidgin est écarté, bien qu'il eût permis de mieux rallier les anglophones, qui sont peu enclins à s'assimiler à la majorité française et qui ne maitrisent pas non plus l'anglais standard. Le pidgin est la langue du peuple, surtout dans les villes de Douala et Yaoundé, alors que l'anglais est la langue des élites. Toutefois, les années 1980-1990 voient de profonds changements politiques et le pidgin-english devient un moyen d'expression politique des plus importants. Pendant les «années de braise » (1990-1993), les revendications pour des élections libres, la création d’un parti d'opposition et les manifestations publiques mènent le Cameroun au bord de la guerre civile. Rostand attribue un rôle majeur dans cette série d'événements à Fru Ndi, ce leader analphabète, dont le charisme reposait sur une maîtrise parfaite du pidgin, «manié avec dextérité », au point qu'il avait su soulever les populations du nord du pays, traditionnellement réfractaires à ce genre de démonstrations publiques. Durant la même période, un musicien dont on s'arrachait littéralement les disques sur les places de marché, attisait la grogne populaire par ses dénonciations, en pidgin-english, des « réalités sociales, le chômage, la vie chère, et la gabegie des politiciens en place ». Pour Rostand, la réussite de l'un et de l'autre ne tient pas à leur expérience politique, mais bien à ce qu'ils ont réussi à rejoindre francophones comme anglophones, «en utilisant le pidgin-english comme mode de ralliement ».

Tout en proscrivant toujours l'usage du pidgin par des campagnes qui encouragent l'usage de l'anglais au foyer, l'État a bien compris le pouvoir de cette langue lors des années de braise. Depuis, 
de nombreux politiciens anglophones ont troqué l'anglais pour le pidgin dans les campagnes électorales. En revanche, les médias publics ne laissent toujours pas de place à cette langue, ce qui explique en partie leur manque de popularité. En fait, le pidgin-english est devenu un outil de contestation, de dissidence, de critique du pouvoir, qui rallie toujours anglophones et francophones. Des artistes dissidents ont été arrêtés pour avoir exprimé leurs vues « dans cette langue que personne n’est supposé comprendre, mais que tout le monde comprend parfaitement ».

Pour Rostand, le pidgin-english est la langue qui aura eu le plus d'influence sur la vie politique et culturelle du Cameroun. La montée en puissance de cette langue soulève la question de sa standardisation, de son écriture, de son enseignement, qui restent à faire si tant est que l'on veuille le faire. L'auteur nous met en garde contre les dangers d'une standardisation qui passerait par l'écrit, risquant ainsi de marginaliser ceux et celles qui n'auront pas fréquenté l'école. Il conclut toutefois en signalant le caractère inéluctable et urgent de reconnaître la langue pour le développement socioéconomique du pays.

Le pidgin-english, qu'on appelle aussi kamtok (de «Cameroon Talk»), est une langue hybride combinant l'anglais et le français (à des degrés divers selon les dialectes) à des substrats africains (Todd et Jumbam 1992). Qu’il ait été choisi comme la langue de la résistance au pouvoir et de la contestation n'est pas étonnant. Outre son pouvoir rassembleur, cette langue est symboliquement aux antithèses des langues du pouvoir au Cameroun, ces grandes langues standardisées que sont le français et l'anglais. Et on peut se demander si la perte de ce rôle symbolique ne serait pas aussi le prix à payer pour un pidgin standardisé.

\section{- Prescriptivisme et patriotisme}

L'idée traditionnelle du prescriptivisme linguistique est associée aux États européens, qui ont développé des mécanismes institutionnels (académies linguistiques, écoles, législations) et des activités systématiques d'aménagement linguistique. L’idée traditionnelle du patriotisme linguistique est celle de la lutte d'une minorité pour ses droits linguistiques dans un contexte de confrontation politique, sociale ou législative. Les réalités contemporaines sont plus compliquées et nous forcent à élargir la portée de ces deux concepts et, surtout, des relations qui les unissent. Dans un contexte de mondialisation, les langues régionales ont acquis un statut plus prestigieux, surtout lorsque l'on tient compte du prestige latent, lié aux valeurs d'identité et de solidarité internes à la communauté. En même temps, elles doivent résister aux facteurs adverses qui menacent la diversité culturelle et linguistique. Le patriotisme régional doit s'imposer devant le patriotisme plus imposant 
correspondant à l'identité nationale, qui doit à son tour s'imposer devant une identité sinon planétaire, au moins largement internationale. En parallèle, le prescriptivisme doit se réinventer : il doit faire tomber en partie les barrières strictes qui permettaient d'identifier la norme centrale à laquelle toutes les variétés linguistiques devaient se mesurer, et incorporer à cette norme les apports d'un nombre grandissant de variétés linguistiques ${ }^{11}$.

Comme plusieurs États issus de la colonisation, le Cameroun présente une image qui correspond partiellement à l'idée traditionnelle que l'on se fait du patriotisme. C'est un patriotisme actif, de revendication, dans un contexte d'opposition directe au pouvoir d'État. Là où on s'éloigne de la conception classique, c'est qu'au Cameroun, la langue n'est pas directement l'objet des contestations ; elle sert d'outil pour toucher et mobiliser la population, et l'amener à revendiquer des droits, non pas linguistiques, mais démocratiques et sociaux.

La France, avec sa tradition de grammairiens et de remarqueurs, son Académie française, son rôle central au sein de l'organisation internationale de la Francophonie, correspond bien à cette vision classique du prescriptivisme que j'ai évoquée plus haut. Pourtant, cela ne simplifie pas nécessairement les rapports entre prescriptivisme et patriotisme. La France est soumise aux mêmes tensions entre identité et légitimité que vit la francophonie un peu partout dans le monde, que ce soit au Canada, dans les Antilles, dans les ex-colonies d'Afrique ou sur son propre territoire politique. Dans ce contexte, la question du positionnement des langues marginales (langues régionales ou immigrantes) est inévitable.

Comme nous l'a montré Hamez, les prescriptions institutionnelles françaises en matière d'éducation ont évolué d'une vision centrée sur le monolinguisme, préconisant l'usage des textes littéraires pour l'apprentissage de la langue, à une vision de l'élève comme locuteur du français langue étrangère, pour aboutir finalement à une position paradoxale où le prescriptivisme français doit faire une place à la vision européenne. Les élèves nouvellement arrivés sont reconnus pleinement comme de non-francophones et l'on fait le vœu pieux de les préparer le mieux possible à l'éducation régulière en français, sans toutefois pallier les difficultés que pose l'enseignement à cette clientèle.

En 2008, la France a finalement reconnu officiellement la légitimité des langues régionales, incluant dans la Constitution un article stipulant que celles-ci «appartiennent au patrimoine de la France ». On peut voir ce changement comme l'ultime résultat de ce long débat parlementaire rapporté par Moliner, mais il n'est pas clair que ce soit là un réel motif de réjouissance. Comme on

\footnotetext{
${ }^{11}$ Voir notamment les travaux de Clyne et Pöll sur les langues pluricentriques.
} 
pouvait s'y attendre, les patriotes concernés n’ont pas réagi de façon unanime à la nouvelle. Si les responsables politiques locaux l'ont généralement bien accueillie, certains n'y voient qu'un début et espèrent des initiatives plus concrètes. Par contre, des indépendantistes polynésiens y voient « une énième attitude colonialiste» qui fait des langues polynésiennes une "propriété de la France» (Ternisien 2008). Je le répète, le patriotisme des uns n’est pas le patriotisme des autres.

Et pourtant, pour l'État français, cette reconnaissance est un pas qui coûte puisque la langue est au cœur de l'identité nationale. Ce n'est pas d'hier que l'État français utilise la langue comme instrument de définition, de cristallisation, de promotion de l'identité nationale, en ignorant toute une partie de sa population qui n'est pas à proprement parler francophone. En effet, il aura fallu attendre le creuset unificateur de la Première Guerre pour voir aboutir le projet révolutionnaire et jacobin de l'abbé Grégoire d'éliminer les patois au profit du français. Et la langue semble absolument nécessaire à cette identité nationale qui, pour certains, « repose d'abord sur un héritage, sur cette possession d'un riche legs de souvenirs » (Lefeuvre 2008 : 152) et non pas sur une identité ethnique clairement circonscrite, dans une France qui « ne contient pas un peuple mais cent » et

«[...] qui n'a été fondée par aucun peuple particulier. Elle porte le nom d'un groupe germanique, parle une langue dérivée du latin, avec un fort accent gaulois nous disent les linguistes. Elle fut inventée par une communauté de peuples. Plus que toute autre nation au monde, elle est un défi vivant aux déterminations ethniques et culturelles. » (Le Bras et Todd $1981: 25)$

D’ailleurs, un sondage paru en 2009 plaçait la langue (80\% des réponses) en tête des «éléments importants qui constituent l'identité en France », devant la République (64\%), le drapeau tricolore (63\%), la laïcité (61\%) et même La Marseillaise (50\%) (Le Parisien 2009).

Accorder des droits linguistiques ou un certain statut à une langue marginalisée est coûteux, c'est vrai, mais comme le note Spolsky, pas autant que d'accorder un statut politique indépendant. Les activistes linguistiques constituent en quelque sorte une «valve de sûreté pour la pression séparatiste» (Spolsky 2009: 205). Le patriotisme peut donc finalement s'avérer fort utile au prescriptivisme.

\section{Pour conclure}

Est-il possible de conclure? Que pourrais-je ajouter qui puisse donner une perspective globale des relations entre prescriptivisme et patriotisme ? Ces relations se jouent sur différents terrains, dont les frontières ne sont pas aussi strictement délimitées que l'organisation de ce numéro le suggère. Le 
décalage entre la norme «objective» et la norme perçue par le sujet parlant, entre l'usage et la perception de l'usage (Partie I) découle des représentations linguistiques et des attitudes, qui sont elles-mêmes liées au prestige légitime et au prestige latent. Les frères ennemis se définissent et se redéfinissent constamment, l'un par rapport à l'autre. Sur le terrain de la vitalité ethnolinguistique et de l'aménagement linguistique (Partie II), les valeurs symboliques associées à la langue (attitudes, représentations, prestiges) constituent des facteurs déterminants de l'avenir de la langue. Enfin, sur le terrain des actions patriotiques et prescriptives explicites (Partie III), les relations entre les deux frères ennemis révèlent encore une fois la mouvance des identités de l'un et de l'autre, construites tantôt avec l'autre, tantôt contre l'autre.

Les articles de ce numéro défrichent une bonne partie du terrain des relations entre prescriptivisme et patriotisme. Pris dans leur ensemble, ils permettent d'abattre ces arbres qui nous cachent la forêt. Plus on défriche, plus on est en mesure de voir l'étendue de la forêt vierge qui s'étend encore devant nous et plus on est à même d'apprécier la riche diversité des écosystèmes linguistiques.

\section{Références bibliographiques}

Blommaert, Jan. 2005. Discourse. Cambridge : Cambridge University Press.

Blommaert, Jan. 2010. The sociolinguistics of globalization. Cambridge : Cambridge University Press.

Bouchard, Chantal. 1998. La langue et le nombril. Histoire d'une obsession québécoise. Montréal : Fides.

Bourdieu, Pierre. 1982. Ce que parler veut dire. L’économie des échanges linguistiques. Paris : Fayard.

Cajolet-Laganière, Hélène et Pierre Martel. 1996. Le français québécois : une langue à plusieurs visages. Sommet 9 (2) :1-3. http://www.usherbrooke.ca/sommets/v09/n2/visage.htm

Charaudeau, Patrick. 2009. Identité sociale et identité discursive. Un jeu de miroir fondateur de l'activité langagière. Dans Identités sociales et discursives du sujet parlant, sous la direction de Patrick Charaudeau, 15-28. Paris : L'Harmattan.

Clyne, Michael (dir.). 1992. Pluricentric languages: Differing norms in different nations. Berlin : Mouton de Gruyter.

Dorian, Nancy. 1981. Language death. Philadelphia : University of Pennsylvania Press.

Dorian, Nancy (dir.). 1989. Investigating obsolescence. Cambridge : Cambridge University Press.

Ferguson, Charles A. 1959. Diglossia. Word 15: 325-340.

Fishman, Joshua. 1967. Bilingualism with and without diglossia: Diglossia with and without bilingualism. Journal of Social Issues 32 (2) : 29-38.

Fishman, Joshua. 1972. Language and nationalism: Two integrative essays. Rowley : Newbury House. 
Gadet, Françoise. 2003. La V ariation sociale en français. Paris : Ophrys.

Garmadi, Juliette. 1981. La sociolinguistique. Paris : Presses universitaires de France.

Giles, Howard, Richard Y. Bourhis et Donald M. Taylor. 1977. Towards a theory of language in ethnic group relations. Dans Language, ethnicity, and intergroup relations, sous la direction de Howard Giles, 307-348. New York : Academic Press.

Hagège, Claude. 2000. Halte à la mort des langues. Paris : Odile Jacob.

Haugen, Einar. 1972. The ecology of language. Stanford : Stanford University Press.

Jones, Mari C. 1998. Language obsolescence and revitalization. Oxford : Clarendon Press.

Krauss, Michael. 1991. The world's languages in crisis. Language $68: 4-10$.

Labov, William. 1966. The social stratification of English in New York City. Washington: Center for Applied Linguistics.

Lambert, Wallace E. 1967. A social psychology of bilingualism, Journal of Social Issues 21 : 91-108.

Le Bras, Hervé et Emmanuel Todd. 1981. L'invention de la France. Paris : Hachette.

Lefeuvre, Daniel. 2008. Faut-il avoir honte de l'identité nationale? Paris : Larousse.

Le Parisien. 2009. Identité nationale : 60 \% des Français favorables au débat, Le Parisien, 31 octobre 2009. http://www.leparisien.fr/politique/identite-nationale-60-des-francais-favorables-audebat-31-10-2009-694943.php.

Mackey, William F. 1976. Bilinguisme et contact des langues. Paris : Klincksieck.

Meillet, Antoine. 1926. Linguistique bistorique et linguistique générale. $2^{\mathrm{e}}$ édition. Paris: Honoré Champion.

Moreau, Marie-Louise. 1999. Pluralité des normes dans la francophonie. DiversCité Langues. http://www.teluq.uquebec.ca/diverscite.

Pöll, Bernhard. 2005. Le français langue pluricentrique? Études sur la variation diatopique d'une langue standard. Frankfurt : Peter Lang.

Remysen, Wim. 2010. La politique linguistique des médias publics au Québec et en Flandre: de quelle conception de la langue est-il question ? Dans Hétérogénéité et homogénéité dans les pratiques langagières : mélanges offerts à Denise Deshaies, sous la direction de Wim Remysen et Diane Vincent, 115-150. Québec : Presses de l'Université Laval.

Spolsky, Bernard. 2009. Language management. Cambridge : Cambridge University Press.

Ternisien, Xavier. 2008. L'entrée des langues régionales dans la Constitution suscite des espoirs, Le Monde, 1er août 2008. http://www.lemonde.fr/societe/article/2008/07/31/1-entree-deslangues-regionales-dans-la-constitution-suscite-des-espoirs_1079043_3224.html

Todd, Loreto et Martin Jumbam. 1992. Kamtok : Anatomy of a pidgin. English Today 8 : 3-11.

Trudgill, Peter. 1972. Sex, covert prestige and linguistic change in the Urban British English of Norwich. Language in Society 1 (2): 175-195.

UNESCO. 2005. Convention sur la protection et la promotion de la diversité des expressions culturelles. Paris.

Vézina, Robert. 2009. La question de la norme linguistique. Québec: Conseil supérieur de la langue française. 